\title{
Coulisses
}

Revue de théâtre

40 | Hiver 2010

Jeux de rappels chez Marivaux

\section{Le dire sans mots dans la tragédie Annibal de Marivaux}

Diana-Adriana Lefter

\section{(2) OpenEdition}

1 Journals

Édition électronique

URL : https://journals.openedition.org/coulisses/662

DOI : 10.4000/coulisses.662

ISSN : 2546-9460

Éditeur

Presses universitaires de Franche-Comté

Édition imprimée

Date de publication : 1 janvier 2010

Pagination : 83-96

ISBN : 978-2-84867-283-0

ISSN : $1150-594 X$

\section{Référence électronique}

Diana-Adriana Lefter, "Le dire sans mots dans la tragédie Annibal de Marivaux », Coulisses [En ligne], 40 | Hiver 2010, mis en ligne le 30 novembre 2016, consulté le 29 décembre 2022. URL : http://

journals.openedition.org/coulisses/662 ; DOI : https://doi.org/10.4000/coulisses.662

Ce document a été généré automatiquement le 29 décembre 2022.

Tous droits réservés 


\title{
Le dire sans mots dans la tragédie Annibal de Marivaux
}

\author{
Diana-Adriana Lefter
}

1 Dans un contexte littéraire qui avait déjà vu naître plusieurs tragédies inspirées de la mort du général carthaginois Annibal ${ }^{1}$, Marivaux choisit le même personnage historique pour héros central de sa seule tragédie, représentée pour la première fois en 1720. Fidèle à la dramaturgie classique qui imposait que les personnages historiques ne soient pas trop déformés, Marivaux surprend dans sa pièce de théâtre la partie finale de la vie d'Annibal, son refuge à Nicomédie, et présente le général comme un vieux guerrier digne, qui n'a perdu ni le mépris des Romains, ni la générosité du cœur.

Bien que la parole soit l'élément fondamental dans une pièce de théâtre, permettant la progression et la compréhension de celle-ci, le silence peut aussi contribuer à la complétude du message, le silence et la parole devenant complémentaires. D'ailleurs, pour Maurice Maeterlinck, «les paroles que nous prononçons n'ont de sens que grâce au silence où elles baignent ${ }^{2}$ ». Cela implique que le silence est une dimension du langage, loin d'être sa négation.

3 Selon Arnaud Rykner en outre, le silence est le vide qui « suit» ou « aspire $»^{3}$ les mots, sans - ajouterions-nous - en détruire le message. Il y a, comme l'affirmait Maeterlinck dans son ouvrage dédié au silence théâtral, un "silence passif», qui est la simple absence de langage et un "silence actif », une sorte de taire c'est faire, qui est " éveillé » et « intronisée » par une circonstance inattendue :

«Le silence est l'élément dans lequel se forment les grandes choses, pour qu'enfin elles puissent émerger, parfaites et majestueuses, à la lumière de la vie qu'elles vont dominer ${ }^{5} »$.

4 Nous nous proposons d'analyser dans ce qui suit ce que disent les personnages de cette tragédie par leurs silences, la manière par laquelle le silence, les mots tus ou les gestes, complètent ou soutiennent le message transmis par les mots. Il y a des silences qui ponctuent les dialogues entre les nobles et leurs confidents, entre les nobles, entre les amoureux, aussi bien que des gestes qui remplacent les mots tout en transmettant un message. Ces silences surviennent dans les moments-clés, de grande tension 
dramatique, et ont le plus souvent pour rôle de garder la face ${ }^{6}$ des interlocuteurs, en cachant leur lâcheté ou en soulignant leur noblesse.

5 La spécificité de la parole théâtrale est que, dans le théâtre, la parole est action, ou en paraphrasant Ducrot, dans le théâtre, dire c'est faire. Pourtant, il est tout aussi vrai que le silence dans le théâtre peut aussi faire ou faire de plus, dans le sens où le message transmis par le code non-verbal peut être plus significatif que celui transmis par le code verbal.

6 Si l'abbé d'Aubignac proclamait pour les classiques la primauté de la parole et lui accordait le rôle premier dans la transmission du message, car « le poète s'explique par la bouche des acteurs : il n'y peut employer d'autres moyens ${ }^{7}$ ", Marivaux se montre souvent novateur dans le dialogue dramatique, en construisant le message à travers les paroles dites, mais aussi à travers les gestes et les silences, car "le langage parlé perceptible à l'oreille s'accompagne normalement de mouvements visibles ${ }^{8}$ ».

7 À des moments-clés du déroulement dramatique dans Annibal, le message n'est pas transmis par des paroles, mais par des éléments para-verbaux: le silence, qui s'actualise dans des interruptions, dans des réticences et dans des énoncés inachevés ${ }^{9}$ et par les «jeux de la physionomie ${ }^{10} »$, notamment par des larmes qui remplacent la parole et qui viennent transmettre un message.

8 Ces suspensions du flux verbal que sont les silences et les jeux de la physionomie n'entraînent pas, dans la pièce de Marivaux, un décodage fautif du message. Le contexte situationnel, les présupposés sociaux et relationnels ${ }^{11}$ que les participants à l'échange conversationnel partagent et même établissent fonctionnent comme des filtres, ayant comme résultat un décodage correct et complet du message transmis. Se poserait alors la question du choix: pourquoi, dans des moments d'intensité dramatique extrême, le message n'est-il pas transmis à travers les paroles ? C'est, selon nous, le choix de la pudeur et du bon sens : ce qui porte atteinte à la dignité, au code comportemental, aux valeurs de classe partagées, au bon sens tout court, ne saurait pas être prononcé. Parce que, «[...] le sens commun, tel que Marivaux le décrit, paraît comprendre comme naturelles des notions du juste et de l'injuste. Il présuppose, en particulier, la sociabilité comme une affection propre à l'homme ${ }^{12}$ ».

\section{Les jeux de la physionomie - les larmes}

Les larmes qui remplacent la manifestation verbale des personnages apparaissent trois fois dans la tragédie de Marivaux et sont les manifestations « discursives » de Laodice.

Dans l'acte IV, scène II, cette manifestation physique est annoncée par le discours verbal de la princesse; ces larmes qui n'apparaissent pas à ce moment comme faire mais comme dire ont un rôle d'anticipation. Ayant deviné le motif de la présence de Flaminius à la cour du roi, aussi bien que l'attitude que Prusias compte adopter à cet égard, Annibal choisit déjà le suicide: il préfère renoncer à la vie que perdre son honneur en devenant esclave des Romains. Mais, pour confirmer ses soupçons, il consulte Laodice, dont l'honneur, la générosité et la foi sont hors de doute. C'est le premier moment où la princesse anticipe cette manifestation : elle annonce qu'elle va verser des larmes pour annoncer à Annibal la trahison dont il serait victime :

ANNIBAL

Parlez, ne craignez point que ma bouche trahisse 
La faveur que ma gloire attend de Laodice.

Quel est donc cet époux que l'on vient de vous offrir?

Puis-je vivre, ou faut-il me hâter de mourir?

LAODICE

Vivez, seigneur, vivez ; j'estime trop moi-même

Et la gloire et le cœur de ce héros qui m'aime

Pour ne l'instruire pas, si jamais dans ces lieux

Quelqu'un lui réservait un sort injurieux.

Oui, puisque c'est à moi que ce héros se livre,

Et qu'enfin c'est pour lui que j'ai juré de vivre,

Vous devez être sûr qu'un cœur tel que le mien

Prendra ses sentiments qui conviennent au sien ;

Et que, me conformant à votre grand courage,

Si vous deviez, seigneur, essuyer un outrage,

Et que la seule mort pût vous en garantir,

Mes larmes couleraient pour vous en avertir. (25)

11 Dans cette première occurrence, les larmes sont, comme nous l'avons déjà dit, présentes dans le discours et non pas dans la manifestation de la physionomie. L'anticipation du pleur apparaît comme une réponse à une demande exprimée par le code verbal : "Parlez », dit Annibal, dans un impératif qui veut exclure de la réponse attendue toute trace de ménagement, de pitié ou de détour. De plus, la demande de verbalisation est soutenue par la promesse du silence: l'aveu de la princesse n'entraînerait pas une réaction verbale de la part d'Annibal; mais un silence dans lequel l'action - le suicide dans son cas - serait la réponse.

Pour expliquer le choix de Laodice, d'annoncer par des larmes et non pas par des paroles le message qu'elle aura pour celui qui devrait devenir son époux, il faudrait interroger le contexte situationnel, les présupposés sociaux et relationnels qui s'établissent entre les deux personnages: Annibal est, comme il vient de le réitérer devant Laodice, un ancien guerrier, un héros, vainqueur autrefois des Romains, mais se trouvant maintenant exilé à Nicomédie, à la cour et sous la protection de Prusias :

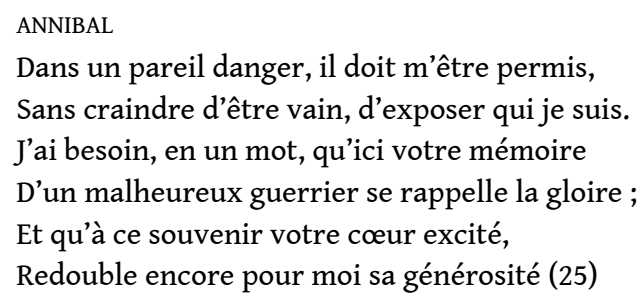

Pourtant, Annibal n'est pas seulement l'hôte et le pair du roi Prusias, il est aussi l'époux promis à Laodice. Ainsi, le vieux guerrier se trouve avec la princesse dans un rapport institutionnel : ancien chef d'armée - princesse, mais aussi privé : futur époux. Dans ce contexte, le pleur de Laodice revêt une dimension sociale et individuelle dans le même temps.

$\mathrm{Du}$ point de vue social, Laodice annonce la réitération d'une pratique du pleur spécifique à la zone méditerranéenne: il s'agit de la figure de la pleureuse, qui est chargée, selon la tradition de cette aire géographique, de manifester par ce rituel codifié, la douleur et le deuil. Ainsi, le message transmis par le pleur de Laodice sera le deuil qui lui sera imposé par la mort d'Annibal. De plus, et toujours dans la sphère sociale, les larmes de Laodice auront une fonction cathartique: elles serviront à 
l'expurgation de la honte qu'elle ressentira en devant être témoin de la trahison de son père.

Dans la sphère privée, les larmes de Laodice pourraient être rapprochées des larmes de l'amante, qui pleure, dans ce cas, non pas tellement l'amour perdu - on sait que la passion amoureuse de Laodice est pour Flaminius - mais un amour digne de son rang et auquel elle a consacré toute sa foi. D'ailleurs, le décodage que fait Annibal des larmes que Laodice versera est tout à fait correct, puisqu'il connait parfaitement la situation de communication: les larmes que Laodice versera seront dans une égale mesure celles d'une femme qui pleure son amour perdu et celles d'une princesse qui regrette son honneur perdu à cause du geste vil de son père et roi.

ANNIBAL

Madame, honorez-moi de cette confidence.

Parlez-moi sans détour : content d'être estimé,

Je me connais trop bien pour vouloir être aimé.

LAODICE

C'est à vous cependant que je dois ma tendresse.

ANNIBAL

[...] Il ne m'importe plus d'être informé, madame,

Du reste des secrets que j'ai lu dans votre âme ;

Et ce serait ici fatiguer votre cœur

Que de lui demander le nom de son vainqueur.

Non, vous m'avez tout dit en gardant le silence,

Et je n'ai pas besoin de cette confidence. (25-26)

Encore une fois le silence transmet un message tout aussi clair que les paroles. Laodice tait le nom de celui que son cœur a choisi et Annibal comprend dans ce silence la honte de la princesse qui ne peut lui avouer que l'élu de son cœur est un homme qu'Annibal considère un misérable ennemi, un Romain

Dans la scène IV de l'acte final et aussi à l'acte IX, les larmes de Laodice ne sont plus un dire, mais un faire. Ayant compris la lâcheté de son père et la trahison d'Hiéron, Laodice se présente en larmes devant Annibal. C'est la relation sociale, le code de l'honneur et la sincérité du bon sens qu'expriment les larmes de Laodice.

ANNIBAL

$\mathrm{Ah}$ ! c'est vous, généreuse princesse.

Vous pleurez : votre cœur accomplit sa promesse.

Les voilà donc ces pleurs, mon unique secours,

Qui devraient m'avertir du péril que je cours!

LAODICE

Oui, je vous rends enfin ce funeste service. (29)

[...]

FLAMINIUS

Je l'avais pressenti :

Du héros désarmé c'est le dernier parti.

Ah! souffrez qu'un Romain, dont l'estime est sincère,

Regrette ici l'honneur que vous pouviez lui faire.

Le roi s'avance ; ô ciel ! sa fille en pleurs le suit. (31)

En pleurant en public, Laodice ne se montre pas une femme faible, cherchant à exciter la pitié, mais elle fait un geste politique. Les larmes sont donc des larmes politiques, 
d'une sincérité politique qu'elle a choisie comme mode de manifestation digne de son statut royal. Cette idée de la moralité et de la sincérité dans les actions politiques avait été énoncée par Shaftesbury dans Sensus communis ou Essai sur l'usage de la raillerie :

En langage commun aussi bien que selon le sens commun, l'honnêteté est la meilleure politique : mais par un raffinement de la raison, les seuls habiles et bien avisés selon le monde, sont de parfaits scélérats. [...] Un homme ordinaire parlant d'une vilaine action selon les idées du sens commun, dit naturellement et de tout son cœur, qu'il ne voudrait point faire une telle chose pour tous les biens du monde. Mais les gens accoutumés à des spéculations délicates trouvent dans ce cas-là de grandes modifications, quantité de subterfuges, de remèdes et d'adoucissements ${ }^{13}$. théâtre des Lumières apporte une contribution intéressante dans la signification du silence. Plusieurs moments de silence, mais non pas de vide communicationnel, sont repérables dans les dialogues entre Annibal et son confident Amilcar, entre Flaminius et Flavius, entre Prusias et Hiéron. une expression des présupposés relationnels entre les participants à l'acte conversationnel. Certes, le confident n'est pas un simple valet, mais une personne qui entretient avec son maître un rapport de confidence/confiance lui permettant de connaître ses pensées secrètes, ses passions ou ses angoisses; pourtant, tout proche qu'il puisse être de son maitre, le confident n'en est pas moins une personne qui se trouve dans un rapport d'infériorité avec lui. Ce rapport d'infériorité impose certaines restrictions dans le discours du confident: le confident peut suggérer, peut être sincère, peut même dire ouvertement à son maître des vérités, mais il ne peut pas lui imposer une attitude. C'est alors qu'intervient le silence, voué à transmettre justement l'opinion du conseiller qui ne peut pas être verbalisée. demeurant à la cour de Prusias, lequel est supposé être un traître :

AMILCAR

Pendant que de ces lieux la retraite est facile,

En croirez-vous ? Fuyez un dangereux asile ;

Sans attendre ici...

ANNIBAL

Nomme-moi des Etats

Plus sûrs pour Annibal que celui de Prusias. (17) 

les relations d'égalité : dans cette catégorie on peut intégrer les dialogues entre Annibal et Prusias, Flaminius et Prusias, Flaminius et Annibal. Les silences dans ces dialogues peuvent être le résultat de l'estime qu'ils se portent les uns aux autres, du respect - ce qui conduit à des silences voués à ménager la face du récepteur du message ou, au contraire, à lui exprimer le mépris ou le dédain.

À l'acte I, scène III, on assiste à la confrontation entre Flaminius et Prusias. L'ambassadeur Romain est venu à Nicomédie reprocher à Prusias, au nom du Sénat, d'avoir reçu Annibal à sa cour. Le dialogue, qui prend la forme d'une confrontation, met face à face deux personnes qui n'acceptent pas la relation sociale qu'elles partagent: Prusias est roi, tandis que Flaminius n'est qu'un ambassadeur. Ainsi, du point de vue de 
Prusias, Flaminius se trouve en position d'infériorité ; d'autre part, Flaminius se pose en représentant légitime d'un état dominant et conquérant, Rome, et il ne parle pas en son nom, mais au nom de la plus haute instance de cet état, le Sénat. D'ailleurs, le discours que Flaminius fait n'est pas personnel, mais assumé comme officiel : à travers la voix de Flaminius, c'est le Sénat qui parle; or, le Sénat romain ne saurait être inférieur à quelque roi que ce soit. La position des deux tenants de la parole est, dirions-nous, de supériorité non-partagée :

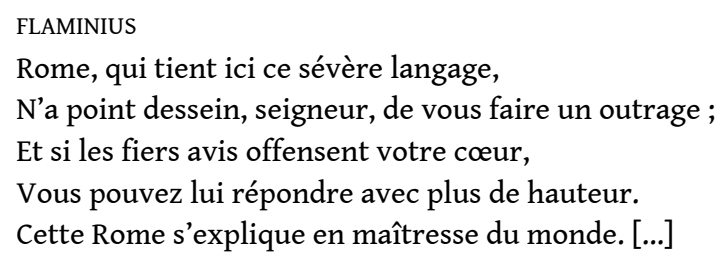

31 Au sein de cette confrontation, Flaminius apporte un nouvel élément : Rome a destiné à la princesse Laodice un époux romain; ce mariage la transformerait, à son tour, en citoyenne romaine. Or, cet époux est Flaminius lui-même, qui aime profondément la princesse. Pourtant, l'ambassadeur romain choisit le silence au lieu de l'aveu devant le roi : ce silence jette une ambiguité qui ne peut être décodée que par le lecteur qui connaît les sentiments de Flaminius. Ce «moi-même» avec lequel il commence la phrase que Prusias interrompt, désigne celui qui aime la princesse, celui destiné par Rome à l'épouser, mais qui hésite devant le roi ; le même mot désigne pour Prusias l'instance qui assume le message émis. Preuve en est la réplique du roi : il permet à l'ambassadeur romain d'annoncer à Laodice le plan que Rome a pour elle :

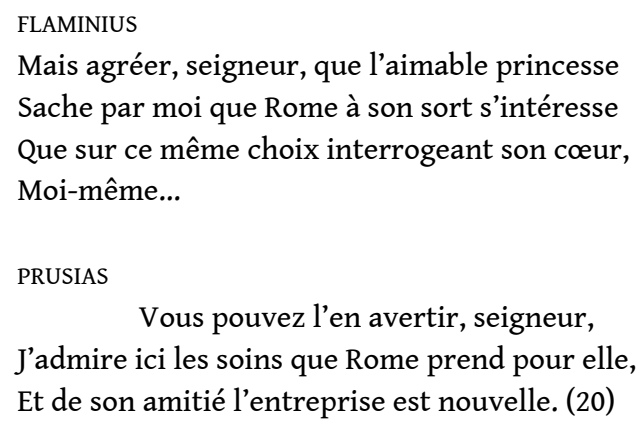

La pensée de Flaminius n'est pas complètement exprimée et son discours reste inachevé surtout à cause de l'hésitation de l'ambassadeur romain. Bien que représentant d'une nation conquérante, Flaminius entend respecter le statut royal de celui qui pourrait devenir son beau-père et manifeste une réticence devant l'aveu complet de ses intentions matrimoniales avec Laodice.

Une autre confrontation entre le roi Prusias et Flaminius se produit à l'acte IV, scène VII. C'est une rencontre courte, pendant laquelle Flaminius comprend que Prusias a décidé livrer Annibal aux mains des Romains, tandis que Prusias n'ose encore le dire ouvertement. Les deux parsèment de silences leurs discours.

Scène VI - PRUSIAS, ANNIBAL, FLAMINIUS 
PRUSIAS

Ici ? n'y craignez rien, seigneur.

ANNIBAL

Je me retire

C'en est assez; voilà ce que j'avais à dire.

Scène VII - FLAMINIUS, PRUSIAS

FLAMINIUS

Ce que dans ce moment vous avez répondu,

M'apprend trop qu'il est temps...

PRUSIAS

J'ai fait ce que j'ai dû...

Arrêtez, le Sénat n'aurait point à se plaindre.

FLAMINIUS

Eh! comment Annibal n'a-t-il plus rien à craindre?

Que pensez-vous?

PRUSIAS

Seigneur, je ne m'explique pas ;

Mais vous serez bientôt content de Prusias.

Vous devez l'être, au moins. (28)

Le silence de Flaminius exprime son doute et son regret à la fois : il veut laisser le roi parler ouvertement, bien qu'il le soupçonne; dans le même temps, l'homme d'honneur et l'homme généreux qu'il est ne peut retenir son regret vis-à-vis du sort d'Annibal. D'autre part, le silence de Prusias transmet sa lâcheté et son double jeu, parce que le silence introduit l'ambiguïté entre le dire et le faire.

Une autre confrontation royale est celle entre Prusias et Annibal, à l'acte V, scène II. C'est l'entretien pendant lequel Prusias veut dévoiler à Annibal sa décision de le livrer aux mains des Romain, au nom de la responsabilité qu'il a pour son peuple. Les manifestations verbales ou non-verbales des deux, lors de cette rencontre, sont justifiées par le savoir qu'ils partagent ou non, ce qui modifie le rapport de forces dans lequel ils se trouvent: Prusias sait qu'il doit, selon le code de l'honneur, protection à son hôte ; il sait aussi qu'il a promis le livrer aux Romains, ce qui enfreint les exigences de sa position; par contre, il ne sait pas qu'Annibal a déjà deviné son plan. Alors, Prusias hésite devant l'aveu, car il veut garder la face. De son côté, Annibal sait quelles sont les obligations que Prusias a envers lui, comme roi et garant de sa protection; il sait également que Prusias l'a trahi, mais l'honneur ne lui permet pas d'en parler avant celui-ci, lui laissant ainsi l'occasion de l'aveu. Dans ce contexte, Annibal est celui qui laisse parler le roi, tandis que Prusias, bien qu'il commence le discours en annonçant la fin du silence, s'arrête à deux moments de l'aveu : ses silences trahissent l'embarras, la honte, la conscience d'avoir enfreint le code comportemental d'un roi :

PRUSIAS

Enfin voici le temps de rompre le silence. [...]

Je doute qu'à vos yeux Prusias excusable

Ne sera point...

ANNIBAL

Oui, seigneur, vous êtes pardonnable. [...] 
PRUSIAS

Seigneur, je le vois bien, trop coupable à vos yeux...

ANNIBAL

Voilà ce que je puis vous répondre de mieux :

Que voulez-vous m'en croire ? oublions l'un et l'autre

Les serments que mon cœur dut refuser au vôtre. (28-29)

Enfin, le silence apparaît également dans le dialogue final entre Annibal et Flaminius. C'est le seul de tous les dialogues évoqués où les tenants de la parole se trouvent en position de parfaite égalité. Bien qu'ils se soient haïs et détestés, chacun reconnaît en l'autre un égal, un héros digne et courageux, auquel il doit du respect : Annibal avoue à Flaminius son projet de suicide, tandis que Flaminius exprime le profond respect qu'il porte à son ancien ennemi. Dans ce contexte, le silence d'Annibal ne porte pas atteinte à la complétude du message, mais c'est le signe d'un désir d'expier en privé, par le poison, par le suicide donc. Flaminius, qui ne demande plus d'éclaircissements, respecte lui aussi ce désir de réserve :

ANNIBAL

Il ne me restait plus, persécuté du sort,

D'autre asile à choisir que Rome ou la mort.

Mais enfin, c'en est fait ; j'ai cru que la dernière

Avec assez d'honneur finissait ma carrière.

Le secours du poison...

FLAMINIUS

Je l'avais pressenti :

Du héros désarmé c'est le dernier parti.

Ah ! souffrez qu'un Romain, dont l'estime est sincère,

Regrette ici l'honneur que vous pouviez nous faire. (31)

\section{Le silence des amoureux}

37 L'acte III, scène II voit la confrontation politico-amoureuse entre Laodice et Flaminius. L'ambassadeur romain vient annoncer à Laodice que c'est lui le mari que le Sénat romain a choisi pour elle. Mais la digne princesse refuse l'offre du Sénat, ne veut même pas laisser parler Flaminius, car par la foi elle est promise à Annibal.

Le premier silence de Flaminius est imposé par Laodice :

FLAMINIUS

Madame, en sa faveur, que votre âme indulgente

Fasse grâce à l'époux que sa main vous présente.

Celui qu'il a choisi...

LAODICE

Ne dites pas son nom : il n'en est pas besoin.

Je dois beaucoup aux soins où le Sénat s'engage ;

Mais je n'ai pas, seigneur, dessein d'en faire usage. (22)

Dans ce cas, la volonté de parler de Flaminius se heurte à la volonté de Laodice de le faire taire. Pour lui, la communication verbale serait la solution au dilemme : il a deviné que la princesse l'aime et il espère concilier amour et devoir politique avec ce mariage. Par contre, Laodice lui impose ce silence parce qu'il lui serait trop difficile d'accomplir 
son devoir politique - celui de refuser le mari romain et de respecter la promesse faite à Annibal - en sacrifiant son amour. Le silence de Flaminius et l'interdiction posée par Laodice sont significatifs: il a laissé entendre par le silence que c'est lui le mari romain ; elle refuse nettement l'offre de Rome, mais non pas Flaminius, parce qu'elle ne l'a pas laissé parler.

Le même jeu du silence imposé pour ne pas refuser l'homme aimé reparaît plus loin dans le dialogue: le discours de Flaminius devient plus explicite, sans pourtant prononcer le nom du mari choisi. Par contre, Laodice refuse que l'information lui soit communiquée par des paroles; la fonction informative de la parole est remplacée par la fonction informative du silence. Le silence qu'elle impose encore une fois à Flaminius lui confirme l'information qu'elle avait devinée : l'homme qu'elle aime est aussi celui que la politique lui a destiné :

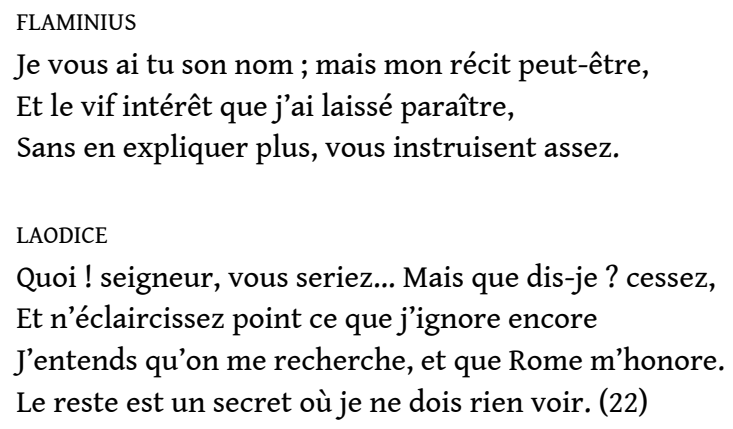

41 La tension dramatique s'accroît dans les stichomythies qui suivent où les silences des amoureux apparaissent comme des stratégies adoptées par les interlocuteurs pour garder la face :

FLAMINIUS

Qu'entends-je ! moi, madame ose m'estimer plus !

N'êtes-vous pas Romaine avec tant de vertus?

Ah ! pourvu que ce cœur partageât ma tendresse...

LAODICE

Non, seigneur ; c'est en vain que le vôtre m'en presse ;

Et quand même l'amour nous unirait tous deux...

\section{FLAMINIUS}

Achevez; qui pourrait m'empêcher d'être heureux!

Vous aurait-on promise? et le roi votre père

Aurait-il...

LAODICE

N'accusez nulle cause étrangère.

Je ne puis vous aimer, seigneur, et vos soupçons

Ne doivent point ailleurs chercher des raisons. (22)

Flaminius exprime par ses silences son espoir en l'amour de Laodice ainsi qu'en son accord pour donné au mariage, aussi bien que sa suspicion : il se doute que l'obstacle à ce mariage serait une promesse faite par le roi à quelqu'un d'autre. De son côté, le silence de Laodice trahit son scepticisme devant le mariage avec Flaminius.

À travers les mots tus, à travers leurs silences, les deux amoureux se montrent dignes de leur statut social ; pour ne pas porter atteinte à la dignité de la femme qu'il aime, Flaminius n'ose pas prononcer le nom du possible mari, ni n'ose jeter le doute sur le roi. 
Quant à Laodice, elle se montre, par les silences qu'elle impose à l'homme qu'elle aime et par ses silences, digne du rôle politique qu'elle assume et auquel elle sacrifie son amour.

Non-dits, sous-entendus, implicites, hésitations, interruptions volontaires ou non du flux verbal, larmes, voilà autant d'actualisations du silence dans le texte théâtral que nous avons étudié. Dès le début de notre travail, nous avons affirmé la complémentarité du silence et de la parole dans le discours théâtral et nous avons également posé comme prémisse le caractère significatif du silence, que nous avons résumé, en paraphrasant Ducrot, par le syntagme quand taire c'est faire.

En inventoriant ensuite les divers types de silences que nous avons repérés dans le texte - larmes, le silence dans la confidence, le silence royal et le silence des amoureux - nous avons pu constater que le silence fonctionne selon les rapports sociaux et relationnels qui s'instaurent entre les personnages. Ainsi, le silence permet aux personnages, dans une égale mesure, de garder la face et, aussi, de ménager la face de l'interlocuteur, tout en transmettant le message que l'on n'ose pas dire dans les paroles.

\section{BIBLIOGRAPHIE}

\section{Texte de référence}

Marivaux, Annibal dans Théâtre complet. Nouvelle édition contenant une pièce non encore recueillie précédée d'une introduction sur la vie et les œuvres de l'auteur par M. Edouard Fournier ornée de vingt magnifiques portraits en couleur par Bertall, Paris, Laplace, Sanchez et Cle, Libraires-Editeurs, 1878. Toutes les références portent sur cette édition.

\section{Bibliographie}

Abée d'Aubignac, La Pratique du théâtre, livre I, chapitre 8, Edition Martino, 1927

Marcel Cohen, Pour une sociologie du langage, Paris, Albin Michel, 1956

Pierre Larthomas, Le Langage dramatique, Paris, PUF, 1980

Maurice Maeterlinck, Le Silence, in Euvres I, Editions Complexe, Bruxelles, 1999

Arnaud Rykner, L'Envers du théâtre, dramaturgie du silence de l'âge classique à Maeterlinck, José Corti, Paris, 1996

Franck Salaün, Pensée de Marivaux, Amsterdam, Rodopi, 2002

Anthony Ashley Cooper Eart of Shaftesbury et Justus van Effen, Essai sur l'usage de la raillerie et de l'enjouement dans les conversations qui roulent sur les Matières les plus importantes, La Haye, chez Henry Scheurleer, MDCCX

Anne Ubersfeld, Lire le théâtre I, Paris, Belin, 1996 


\section{NOTES}

1. Les plus connues tragédies traitant la mort d'Annibal, antérieures à la pièce de Marivaux, sont La Mort d'Annibal de Thomas Corneille, Le Grand Annibal de Georges de Scudéry, Annibal de Nicolas de Motreux et la pièce homonyme de Colonia.

2. Maurice Maeterlinck, Le Silence, in CEuvres I, Éditions Complexe, Bruxelles, 1999, p. 303.

3. Arnaud Rykner, L'Envers du théâtre, dramaturgie du silence de l'âge classique à Maeterlinck, José Corti, Paris, 1996, p. 303.

4. Idem., p. 299.

5. Idem., p. 298.

6. Nous entendons la notion de face dans le sens linguistique, consacré par la théorie des faces de Goffman, comme enjeu fondamental de tout échange. Pour Goffman (Les Rites d'interaction, Paris, Minuit, 1974), la face se définit comme la valeur sociale positive qu'une personne revendique effectivement à travers la ligne d'action que les autres supposent qu'elle a adoptée au cours d'un contact particulier. [...] la face est une image du moi délinée selon certains attributs sociaux approuvés, et néanmoins partageables, puisque, par exemple, on peut donner une bonne image de sa profession ou de sa confession en donnant une bonne image de soi. (p. 9) Pour Goffman, la vie est un théâtre où chaque interlocuteur tente de garder sa face. Cela arrive parce que, lorsque de toute rencontre, la face de l'individu est menacée, d'où son besoin et son désir de la maintenir intacte, de faire bonne figure et d'éviter de perdre la face : La préservation de la face est réalisée en adoptant des attitudes permettant le renvoi à une image cohérente de soi. (p. 11)

7. Abée d'Aubignac, La Pratique du théâtre, livre I, chapitre 8, Edition Martino, 1927, p. 53.

8. Marcel Cohen, Pour une sociologie du langage, Paris, Albin Michel, 1956, p. 68.

9. Pierre Larthomas, Le Langage dramatique, Paris, PUF, 1980.

Larthomas fait une distinction nette entre interruption, réticence et énoncé inachevé, tout en les classifiant de " accidents de langage ». Ainsi, l'interruption est un accident de langage qui ne doit pas être banni parce que sans elle le dialogue perdrait sa vie, tandis que la réticence se manifeste à l'intérieur de la réplique, par une interruption de la phrase, laquelle reste inachevée, mais immédiatement reprise.

10. Pierre Larthomas, op. cit.

11. Anne Ubersfeld, Lire le théâtre I, Paris, Belin, 1996.

12. Franck Salaün, Pensée de Marivaux, Amsterdam, Rodopi, 2002, p. 96.

13. Anthony Ashley Cooper Earl of Shaftesbury et Justus van Effen, Essai sur l'usage de la raillerie et de l'enjouement dans les conversations qui roulent sur les Matières les plus importantes, La Haye, chez Henry Scheurleer, MDCCX, p. 141-142.

\section{INDEX}

oeuvrecitee Annibal 


\section{AUTEURS}

\section{DIANA-ADRIANA LEFTER}

Université de Pitesti, Roumanie, Faculté des Lettres, Département de langues romanes 